\title{
Search for very high energy $\gamma$-ray emission from GRB 190829A with LHAASO-WCDA triggerless data
}

\section{Yuhua Yao, ${ }^{a, b, *}$ Wei Liu, ${ }^{a}$ Bingqiang Qiao, ${ }^{a}$ Yiqing Guo, ${ }^{a}$ Hongbo Hu, ${ }^{a, c}$ Chaowen Yang $^{b}$ and Zhiguo Yao ${ }^{a}$ on behalf of the LHAASO Collaboration}

(a complete list of authors can be found at the end of the proceedings)

${ }^{a}$ College of Physical Science and Technology, Sichuan University, N.29 Wangjiang Road, Jiuyanqiao, Wuhou District, Chengdu City, Sichuan Province, 610064, P.R. China

${ }^{b}$ Institute of High Energy Physics, Chinese Academy of Sciences, No.19B Yuquan Road, Shijingshan District, Beijing, 100049, P.R. China

${ }^{c}$ University of Chinese Academy of Sciences,

19 A Yuquan Rd, Shijingshan District, Beijing 100049, P.R.China

E-mail: yaoyh@ihep.ac.cn

The observation of very high energy (VHE, > 100GeV) photon from $\gamma$-ray bursts (GRBs) can advance our understanding of their radiation mechanism, the evolution of host-galaxies, violations of Lorentz invariance, extragalactic background light, and intergalactic magnetic field. Recently the measurements on VHE emission from afterglow phase have blossomed and borne fruit while detection of the prompt emission is rare. It's important to observe prompt emissions by groundbased instruments which take advantage of wide field of view and high duty cycle. Up to now, of the total six GRBs reported with VHE $\gamma$-ray emissions, GRB 190829A is the nearest one. The detection of its VHE emission was announced by H.E.S.S. at about $4 \mathrm{~h} 20 \mathrm{~m}$ after the burst. While during its prompt phase, GRB 190829A was at the edge of the field of view of LHAASO. Benefiting by the high altitude of $4410 \mathrm{~m}$ and the triggerless observation mode, the energy threshold of LHAASO is largely reduced to tens of GeV. This work presents the search for VHE $\gamma$-ray emission from GRB 190829A with the triggerless data taken by the first pond of LHAASOWCDA. The analysis shows no indication of VHE emission neither in the prompt nor in the first 2 hours of afterglow phase, thus $95 \%$ upper limits are yielded. Furthermore, the upper limits of prompt phase was used to constrain the spectral parameter space of VHE extra component based on a phenomenological model.

$37^{\text {th }}$ International Cosmic Ray Conference (ICRC 2021)

July 12th - 23rd, 2021

Online - Berlin, Germany

\footnotetext{
*Presenter
} 


\section{Introduction}

$\gamma$-ray bursts (GRBs) are the most energetic explosive events occurring in the cosmological distance. Fifties years after their discovery, many aspects of GRB are still subject to heavy debate. Their origin invokes the messy physics related to the progenitor, central engine, jet composition. Various works predict GRB could produce photons in the very-high energy (VHE, > 100GeV) range, even up to $\mathrm{TeV}$ energies [1-4]. The observation of such high energy would provide key information about internal environment, structure of jet, particle acceleration, radiative mechanisms about GRBs, as well as constrains on extragalactic background light (EBL) models and the violation of Lorentz invariance. Compared to the prompt $\gamma$-ray emission, physics about the long-lasting afterglow emission is much simpler and known better, which mainly depend on a jet and a stellar medium.

GRB is a prime objective of ground-based instruments. Of two categories, the imaging atmospheric Cherenkov telescopes (IACTs), limited by their narrow field of view (FOV) and restricted operation conditions, have difficulty in observing the prompt emission from GRBs. Despite this, they do have the advantage in the observation of afterglow emission with their excellent sensitivity. Extensive air shower (EAS) arrays provide larger instantaneous FOV and higher duty cycle, thus are ideal and possible for the observation of prompt emission. Recently, VHE photons from the afterglow phase of several GRBs have been detected [5-11], which brought both new opportunities and challenges for the study of GRBs. However, no unambiguous evidence for the detection of VHE $\gamma$ rays in the prompt phase of GRBs has been seen in many experiments over years of dedicated efforts.

Among six GRBs reported with VHE emission observation by IACTs, GRB 190829A is the closest one, of which red-shift is $\sim 0.0785$. Occurring on 29 August 2019 at 19:55:53 $\left(\mathrm{T}_{0}\right)$, it was followed up by an enormous observational campaign covering a large fraction of the electromagnetic spectrum, including the VHE band afterglow emission measurements from $\mathrm{T}_{0}+4$ hours to +56 hours by H.E.S.S. [12]. GRB 190829A occurred on the edge of the FOV of LHAASO-WCDA when the first pond o LHAASO-WCDA was in the test running operation.

\section{Follow-up to GRB 190829A with LHAASO-WCDA}

Large High Altitude Array Shower Observatory (LHAASO) is a newly-built $\gamma$-ray detector, which is located at about $4410 \mathrm{~m}$ above sea level. Water Cherenkov Detector Array (WCDA) is one of the major parts, consisting of 3120 water cells, totally covering an area of 78,000 $\mathrm{m}^{2}$. Its wide FOV $\sim 2$ sr, and $>90 \%$ uptime allow it to continuously monitor for emissions higher than hundreds of $\mathrm{GeV}$.

In order to further reduce observational energy threshold, the data acquisition (DAQ) system of LHAASO-WCDA adopts a second triggerless mode as a complementary of the main pattern. With the help of external GRB alerts, in the triggerless mode, DAQ would keep all hit information (eg. charge and time) recorded on each fired detector from $\mathrm{T}_{0}-0.5 \mathrm{~h}$ to $\mathrm{T}_{0}+2 \mathrm{~h}$, once the received GRB triggering at $\mathrm{T}_{0}$ is in the FOV of LHAASO-WCDA. 
A quarter of LHAASO-WCDA, comprising 900 water cells, started its operation in April 2019. In March 2021, the full array is in operation. Up to June 2021, over 100 GRBs have been registered and allowed for follow-up observation by LHAASO-WCDA under triggerless mode.

GRB 190829A occurred at a zenith angle of $46^{\circ}$ of a quarter array. The $\sim 2.5$ hours triggerless data collected with the first pond was used to search for high energy emission.

\section{Analysis}

Data collected by LHAASO-WCDA in the triggerless mode consists of event-induced hits and random noise. Usually the latter one is a bottleneck problem for EAS array to search for $\gamma$-ray signals. With the GRB location reported by satellites and concentrated space characteristics of air shower, a new self-triggered method is adopted to search for photons from GRBs, which can largely reduce random noises. As is well-known, secondary particles of an air shower distribute in a thin disk perpendicular to the incident direction of primary particle. As shown in figure 1, the temporal and spatial distribution of hits of a Monte Carlo photon-induced air shower are concentrated, while the noise are almost uniform distribution. Using a narrow temporal and spatial trigger window perpendicular to the source direction, hits of events from (or near to) the direction of the source are more likely to be preserved, a hit clustering is selected as seeds of a shower event. The narrower the self-trigger window is, the more the noise is eliminated. Afterward, more hits are identified by performing a flexible time search near the trigger time, which allows one to finely optimize for the determination of an event. This flexible time window is determined by the time interval of two hits. At last this event is packed and used for reconstruction. All hits of a raw-data file are sorted with time, a sliding time window is used to loop over all hit clustering for candidate events.
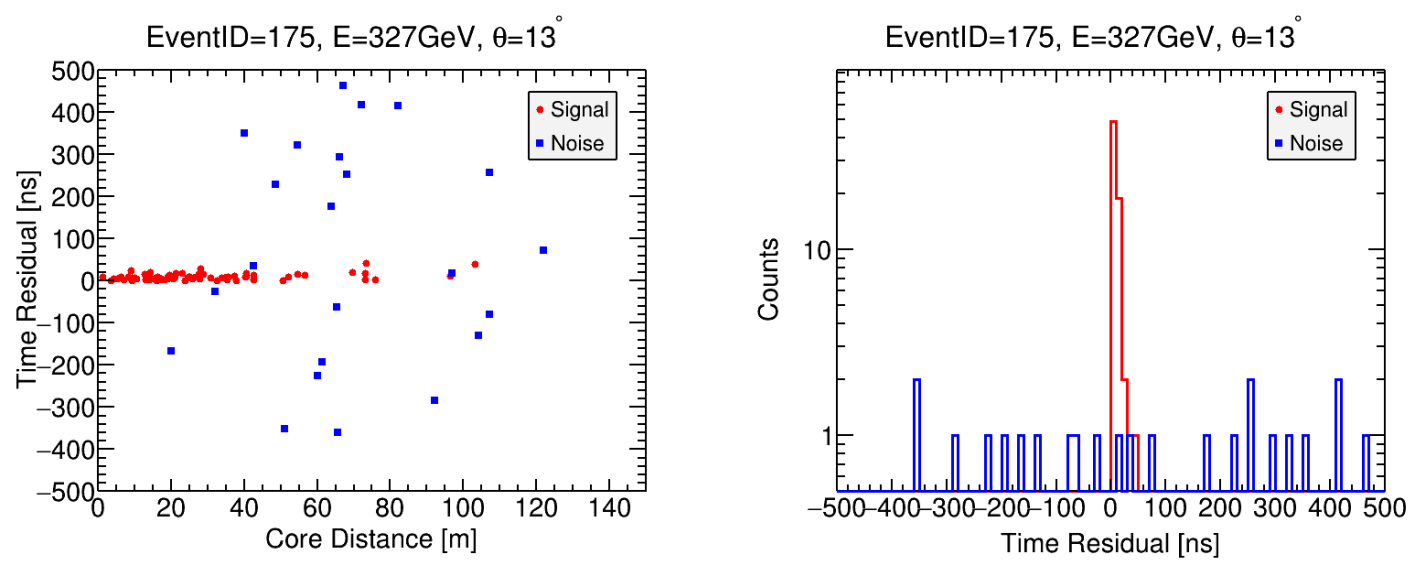

Figure 1: Left, temporal and spatial distribution of hits of a Monte Carlo photon-induced air shower. Right, temporal distribution of this event. Red points are from $\gamma$-ray shower, blue rectangles are random noise.

The performance of a quarter LHAASO-WCDA with above-mentioned method is shown in figure 2. Using a Monte Carlo simulation of the detector response, the angular resolution and the effective area are demonstrated in the left and right, respectively. The former one is used to determine the source-region to exclude events deviating from the source direction, most of these off-source events are all-sky CR backgrounds. 

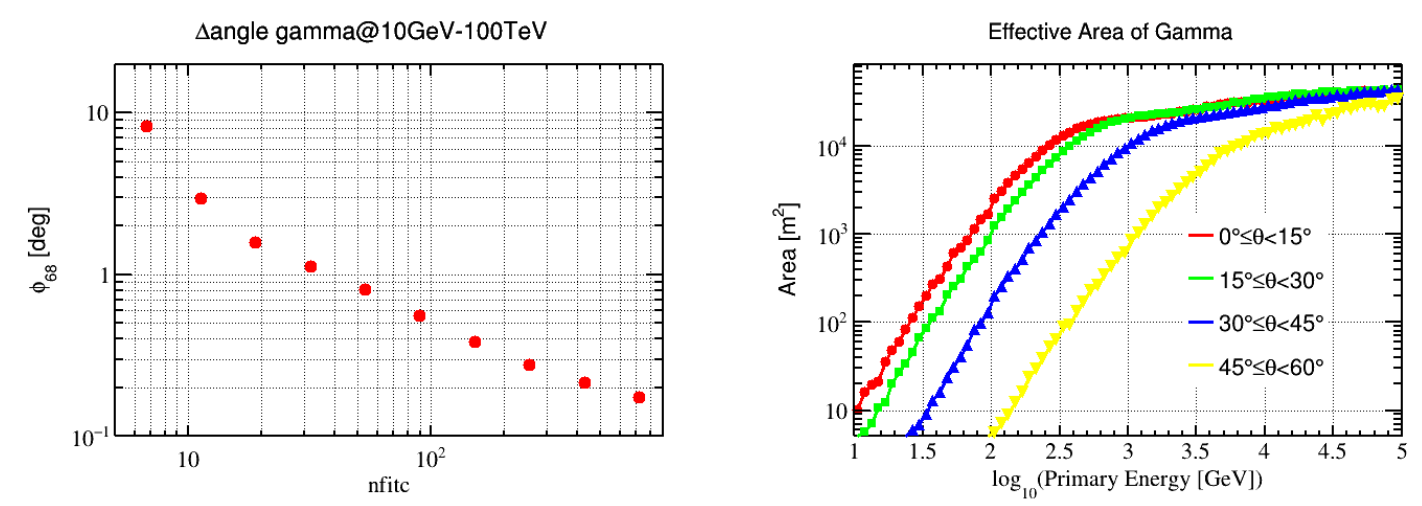

Figure 2: Left, average angular resolution ( $68 \%$ containment radius) at zenith angles $\theta<50^{\circ}$, right, effective area at different zenith angle ranges.

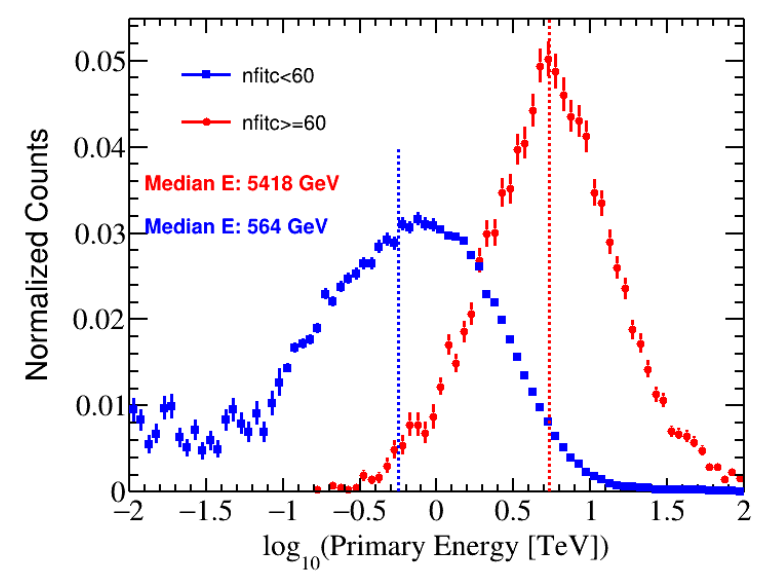

Figure 3: Primary energy dispersive of $\gamma$-ray induced events at nhit $<60$ and nhit $>60$, at zenith angle $40^{\circ}-50^{\circ}$.

In this work, all reconstructed events are divided into hundred $\mathrm{GeV}$ and $\mathrm{TeV}$ energy categories according to the energy estimator nhit $<60$ and $\geq 60$. Figure 3 demonstrates the primary energy distributions of these two samples when the zenith angle in the range of $40^{\circ}-50^{\circ}$. Usually a $\gamma-$ hadron separation parameter $\mathcal{C}$ could be used to further eliminate $\mathrm{CR}$ backgrounds. When primary $\gamma$-ray energy is larger than $\mathrm{TeV} C>15$ is applied.

It is no doubt that there are still CR background contamination in the $\gamma$-like samples in the source region. Two fake sources of equal-declination are adopted to estimate the number of background and signals. These two fake sources are treated with the same process as the true source with the 2.5 hour triggerless data.

\section{Results}

The light curve is one of the most important characteristics of GRBs. The number of $\gamma$-ray events from source direction as a function of time around the $T_{0}$ is showed in Figure 4. No event excess was observed in both energies due to the large fluctuation. Figure 5 presents the significance 
level distributions with time, and statistically obtaining figure 6 . The latter ones are consistent with the Gaussian function.
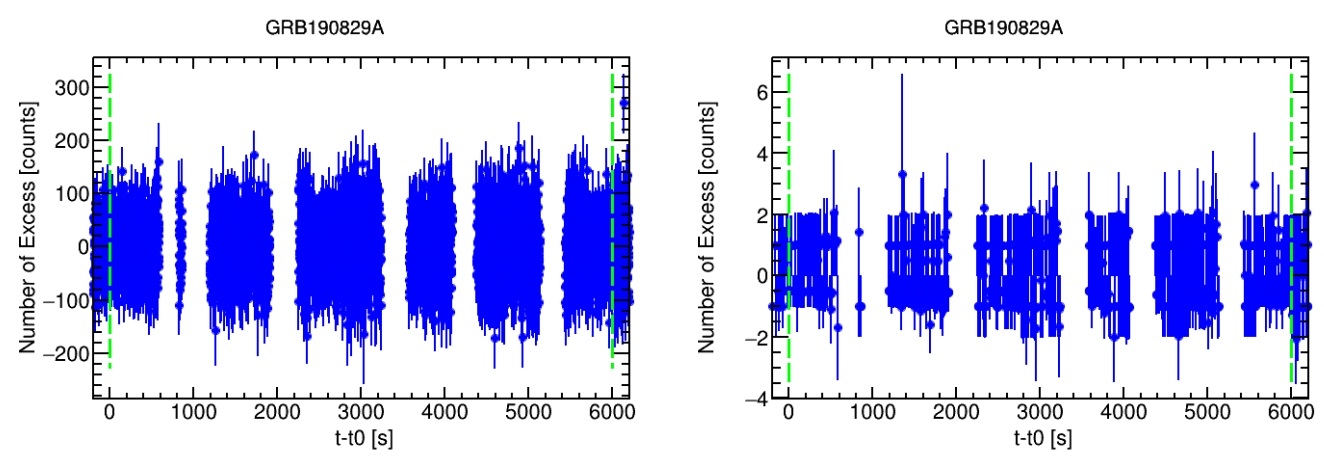

Figure 4: Number of signal candidates distributes with time. Left, nfitc $<60$, right, nfitc $\geq 60$.
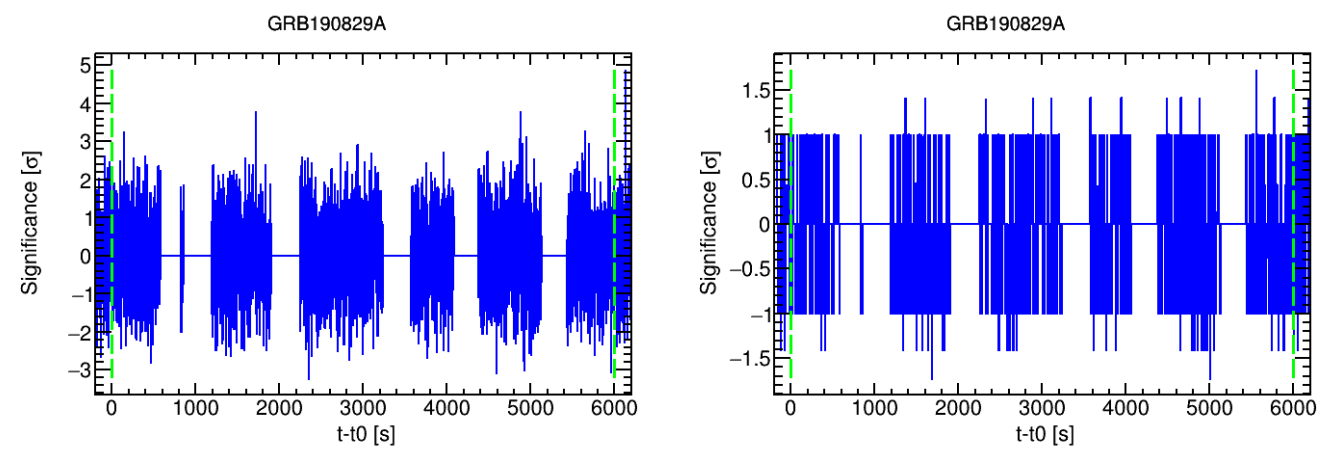

Figure 5: Significance level of signal candidates distributes with time. Left, nfitc $<60$, right, nfitc $\geq 60$.
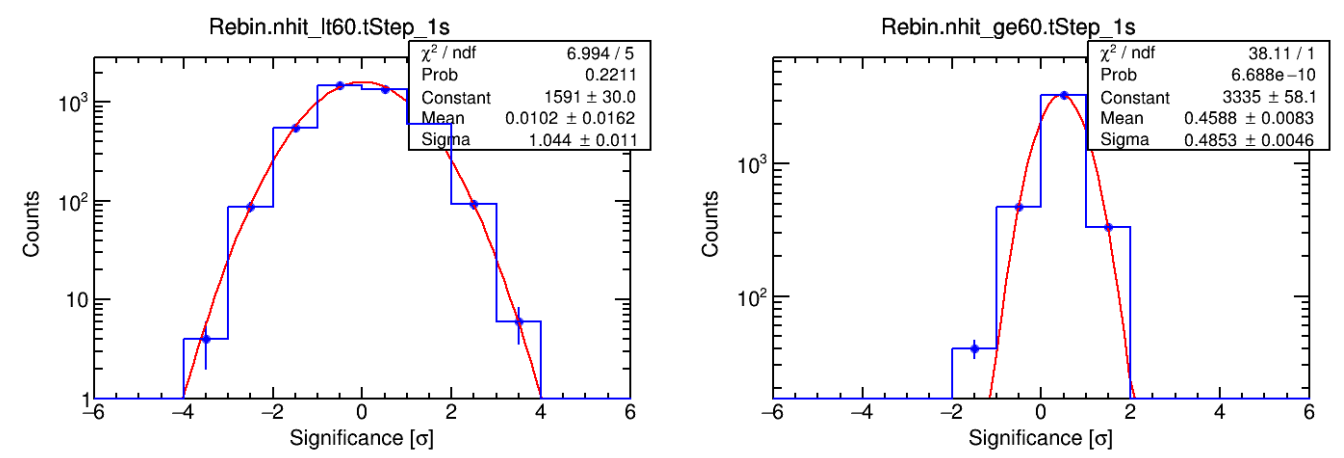

Figure 6: Signal distribution. Left, nfitc $<60$, right, nfitc $\geq 60$.

No significant excess were detected by LHAASO-WCDA, thus $95 \%$ upper limits on the number of signal events are computed following the statistical method given by Helene [13]. By assuming an $\mathrm{E}^{-1.5}$ power-law spectrum for GRB 190829A and considering the EBL absorption effect according to [14], with the detector response, the limit was then convert to flux units for two different energy bands as shown in the left of figure 7. 

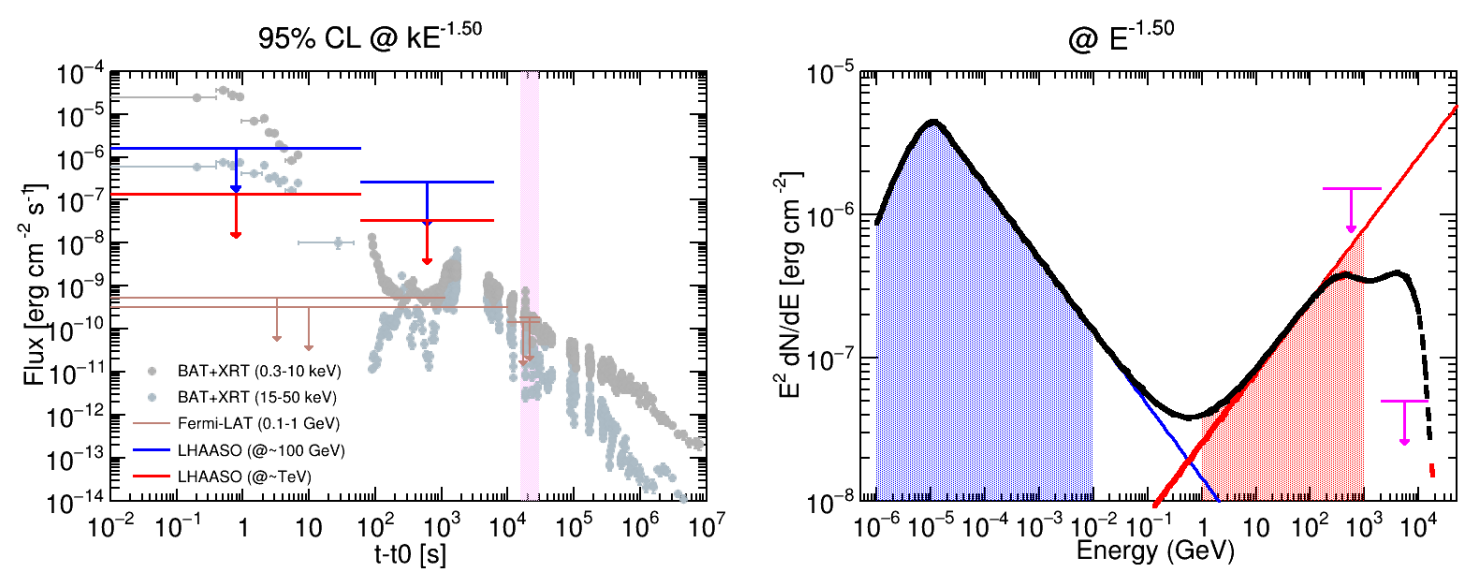

Figure 7: Left, Upper limits from LHAASO. Left, phenomenological spectra model during the prompt phase. Blue and red solid lines represent spectra of Band function and extra component, respectively. Black line is the combination of Band function and extra component, taking the EBL absorption in account.

Though no VHE signal was detected, the flux upper limits can provide important constraint information. It is obvious that the upper limits of prompt phase emission are more useful. During the prompt emission an extra power-law emission was phenomenologically modelled on the top of the Band spectra. Band function parameters are based on the Fermi-GBM, the extra component is described by a pow-law with a index $\beta_{\mathrm{ext}}$ and flux normalization $\mathrm{R}_{\mathrm{ext}}$. Right of figure 7 shows the spectra taking $\mathrm{R}_{\mathrm{ext}}=0.1, \beta_{\mathrm{ext}}=-1.5$. $\mathrm{R}_{\mathrm{ext}}$ represents the ratio of fluences in the energy range $1 \mathrm{GeV}-1 \mathrm{TeV}$ to that of $1 \mathrm{keV}-10 \mathrm{MeV}$, which are the red- and blue-shaded regions, respectively. It can be seen that the TeV upper limit from LHAASO-WCDA is lower than the model-calculated flux, thus it might be used to constrain the magnetic field or some other parameters in a specific detailed model.

\section{Conlucsion}

LHAASO-WCDA is a newly-built $\gamma$-ray array, its wide FOV, high duty cycle, low energy threshold make it suitable for the observation of GRBs. GRB 190829A is the closest GRB, the collected data by a quarter array array of LHAASO-WCDA were used to set upper limits on high energy $\gamma$-ray flux for its prompt phase emission. Furthermore, we simply compared upper limits in the prompt phase with the flux of a phenomenological spectral model. This results would be further used to constrain the magnetic field and other related parameters with detailed simulation works in future.

\section{Acknowledgements}

This work is supported by the Key R\&D Program of Sichuan Province (Grant No. 2019ZYZF0001) and National Natural Science Foundation of China (Grant Nos. 11635011, U1831208). 


\section{References}

[1] Ravi P. Pilla and Abraham Loeb. Emission Spectra from Internal Shocks in Gamma-Ray Burst Sources. ApJ, 494(2):L167-L171, February 1998.

[2] Charles D. Dermer, James Chiang, and Kurt E. Mitman. Beaming, Baryon Loading, and the Synchrotron Self-Compton Component in Gamma-Ray Bursts. ApJ, 537(2):785-795, July 2000 .

[3] Tsvi Piran, Re'em Sari, and Yuan-Chuan Zou. Observational limits on inverse Compton processes in gamma-ray bursts. MNRAS, 393(4):1107-1113, March 2009.

[4] Ž. Bošnjak, F. Daigne, and G. Dubus. Prompt high-energy emission from gamma-ray bursts in the internal shock model. A\&A, 498(3):677-703, May 2009.

[5] MAGIC Collaboration, V. A. Acciari, S. Ansoldi, et al. Observation of inverse Compton emission from a long $\gamma$-ray burst. Nature, 575(7783):459-463, November 2019.

[6] MAGIC Collaboration, V. A. Acciari, S. Ansoldi, et al. Teraelectronvolt emission from the $\gamma$-ray burst GRB 190114C. Nature, 575(7783):455-458, November 2019.

[7] H. Abdalla, R. Adam, F. Aharonian, et al. A very-high-energy component deep in the $\gamma$-ray burst afterglow. Nature, 575(7783):464-467, November 2019.

[8] M. de Naurois. GRB190829A: Detection of VHE gamma-ray emission with H.E.S.S. The Astronomer's Telegram, 13052:1, August 2019.

[9] C. Malacaria, P. Veres, C. Meegan, E. Bissaldi, and Fermi GBM Team. GRB 201216C: Fermi GBM detection. GRB Coordinates Network, 29073:1, December 2020.

[10] MAGIC Collaboration, V. A. Acciari, S. Ansoldi, et al. MAGIC observations of the nearby short gamma-ray burst GRB 160821B. arXiv e-prints, page arXiv:2012.07193, December 2020 .

[11] O. Blanch, M. Gaug, K. Noda, et al. MAGIC observations of GRB 201015A: hint of very high energy gamma-ray signal. GRB Coordinates Network, 28659:1, October 2020.

[12] H.E.S.S. Collaboration, H. Abdalla, F. Aharonian, et al. Revealing x-ray and gamma ray temporal and spectral similarities in the grb 190829a afterglow. Science, 372(6546):1081$1085,2021$.

[13] O. Helene. Upper limit of peak area. Nuclear Instruments and Methods in Physics Research, 212(1-3):319-322, July 1983.

[14] Rudy C. Gilmore, Rachel S. Somerville, Joel R. Primack, and Alberto Domínguez. Semianalytic modelling of the extragalactic background light and consequences for extragalactic gamma-ray spectra. MNRAS, 422(4):3189-3207, June 2012. 


\section{Full Authors List: LHAASO Collaboration}

Zhen $\mathrm{Cao}^{1,2,3}$, F. Aharonian ${ }^{4,5}$, Q. An ${ }^{6,7}$, Axikegu ${ }^{8}$, L.X. Bai ${ }^{9}$, Y.X. Bai ${ }^{1,3}$, L.X. Bai ${ }^{9}$, Y.X. Bai ${ }^{1,3}$, Y.W. Bao ${ }^{10}$, D. Bastieri ${ }^{11}$, X.J. Bi $1^{1,2,3}$, Y.J. Bi ${ }^{1,3}$, H. Cai ${ }^{12}$, J.T. Cai ${ }^{11}$, Zhe Cao ${ }^{6,7}$, J. Chang ${ }^{13}$, J.F. Chang ${ }^{1,3,6}$, B.M. Chen ${ }^{14}$, E.S. Chen ${ }^{1,2,3}$, J. Chen ${ }^{9}$, Liang Chen $^{1,2,3}$, Liang Chen ${ }^{15}$, Long Chen ${ }^{8}$, M.J. Chen ${ }^{1,3}$, M.L. Chen ${ }^{1,3,6}$, Q.H. Chen ${ }^{8}$, S.H. Chen ${ }^{1,2,3}$, S.Z. Chen ${ }^{1,3}$, T.L. Chen ${ }^{16}$,X.L. $\mathrm{Chen}^{1,2,3}$, Y. Chen $^{10}$, N. Cheng ${ }^{1,3}$, Y.D. Cheng ${ }^{1,3}$, S.W. Cui ${ }^{14}$, X.H. Cui ${ }^{17}$, Y.D. Cui ${ }^{18}$, B. D'Ettorre Piazzoli1 ${ }^{19}$, B.Z. Dai ${ }^{20}$, H.L. Dai $^{1,3,6}$, Z.G. Dai ${ }^{7}$, Danzengluobu ${ }^{16}$, D. della Volpe ${ }^{21}$, X.J. Dong ${ }^{1,3}$, K.K. Duan ${ }^{13}$, J.H. Fan ${ }^{11}$, Y.Z. Fan $^{13}$, Z.X. Fan ${ }^{1,3}$, J. Fang ${ }^{20}$, K. Fang $^{1,3}$, C.F. Feng ${ }^{22}$, L. Feng ${ }^{13}$, S.H. Feng ${ }^{1,3}$, Y.L. Feng ${ }^{13}$, B. Gao ${ }^{1,3}$, C.D. Gao ${ }^{22}$, L.Q. Gao ${ }^{1,2,3}$, Q. Gao ${ }^{16}$, W. Gao ${ }^{22}$, M.M. Ge ${ }^{20}$, L.S. Geng ${ }^{1,3}$, G.H. Gong ${ }^{23}$, Q.B. Gou ${ }^{1,3}$, M.H. Gu${ }^{1,3,6}$, F.L. Guo ${ }^{15}$, J.G. Guo ${ }^{1,2,3}$, X.L. Guo ${ }^{8}$, Y.Q. Guo ${ }^{1,3}$, Y.Y. Guo ${ }^{1,2,3,13}$, Y.A.

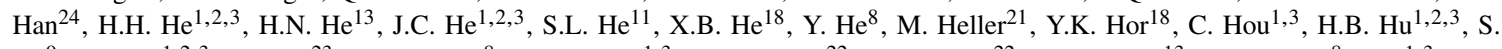

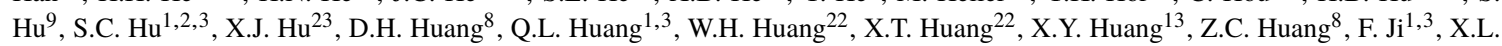
$\mathrm{Ji}^{1,3,6}$, H.Y. Jia ${ }^{8}$, K. Jiang ${ }^{6,7}$, Z.J. Jiang ${ }^{20}$, C. Jin ${ }^{1,2,3}$, T. Ke ${ }^{1,3}$, D. Kuleshov ${ }^{25}$, K. Levochkin ${ }^{25}$, B.B. Li ${ }^{14}$, Cheng Li ${ }^{6,7}$, Cong Li ${ }^{1,3}$,

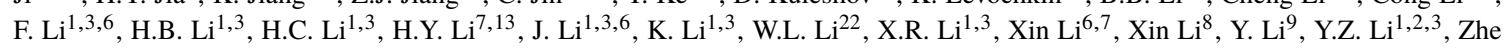

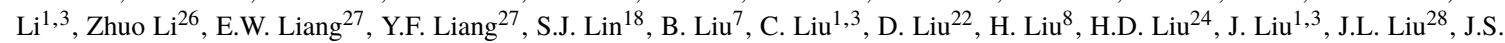

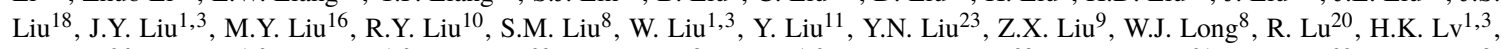

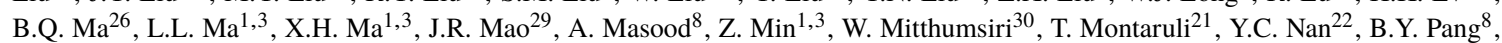
P. Pattarakijwanich ${ }^{30}$, Z.Y. Pei ${ }^{11}$, M.Y. Qi ${ }^{1,3}$, Y.Q. Qi ${ }^{14}$, B.Q. Qiao ${ }^{1,3}$, J.J. Qin ${ }^{7}$, D. Ruffolo ${ }^{30}$, V. Rulev ${ }^{25}$, A. Sáiz ${ }^{30}$, L. Shao ${ }^{14}$, O. Shchegolev $^{25,31}$, X.D. Sheng ${ }^{1,3}$, J.Y. Shi ${ }^{1,3}$, H.C. Song ${ }^{26}$, Yu.V. Stenkin ${ }^{25,31}$, V. Stepanov ${ }^{25}$, Y. Su ${ }^{32}$, Q.N. Sun ${ }^{8}$, X.N. Sun ${ }^{27}$, Z.B.

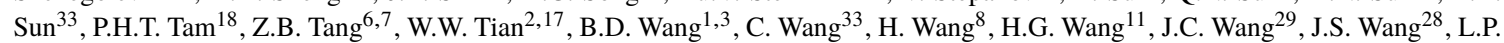
Wang $^{22}$, L.Y. Wang ${ }^{1,3}$, R.N. Wang ${ }^{8}$, W. Wang ${ }^{18}$, W. Wang ${ }^{12}$, X.G. Wang ${ }^{27}$, X.J. Wang ${ }^{1,3}$, X.Y. Wang ${ }^{10}$, Y. Wang ${ }^{8}$, Y.D. Wang ${ }^{1,3}$, Y.J. Wang $^{1,3}$, Y.P. Wang ${ }^{1,2,3}$, Z.H. Wang ${ }^{9}$, Z.X. Wang ${ }^{20}$, Zhen Wang ${ }^{28}$, Zheng Wang ${ }^{1,3,6}{ }^{1, \text { D.M. Wei }}{ }^{13}$, J.J. Wei ${ }^{13}$, Y.J. Wei ${ }^{1,2,3}$, T. Wen $^{20}$, C.Y. Wu ${ }^{1,3}$, H.R. Wu ${ }^{1,3}$, S. Wu ${ }^{1,3}$, W.X. Wu ${ }^{8}$, X.F. Wu ${ }^{13}$, S.Q. Xi ${ }^{1,3}$, J. Xia ${ }^{7}, 13$, J.J. Xia ${ }^{8}$, G.M. Xiang ${ }^{2,15}$, D.X. Xiao ${ }^{16}$, G. Xiao ${ }^{1,3}$, H.B. Xiao ${ }^{11}$, G.G. Xin ${ }^{12}$, Y.L. Xin ${ }^{8}$, Y. Xing ${ }^{15}$, D.L. Xu ${ }^{28}$, R.X. Xu ${ }^{26}$, L. Xue ${ }^{22}$, D.H. Yan ${ }^{29}$, J.Z. Yan ${ }^{13}$, C.W. Yang ${ }^{9}$, F.F. Yang ${ }^{1,3,6}$, J.Y. Yang ${ }^{18}$, L.L. Yang ${ }^{18}$, M.J. Yang ${ }^{1,3}$, R.Z. Yang ${ }^{7}$, S.B. Yang ${ }^{20}$, Y.H. Yao ${ }^{9}$, Z.G. Yao ${ }^{1,3}$, Y.M. Ye ${ }^{23}$, L.Q. Yin ${ }^{1,3}$, N. Yin ${ }^{22}$, X.H. You $^{1,3}$, Z.Y. You ${ }^{1,2,3}$, Y.H. Yu ${ }^{22}$, Q. Yuan ${ }^{13}$, H.D. Zeng ${ }^{13}$, T.X. Zeng ${ }^{1,3,6}$, W. Zeng ${ }^{20}$, Z.K. Zeng ${ }^{1,2,3}$, M. Zha ${ }^{1,3}$, X.X. Zhai ${ }^{1,3}$, B.B. Zhang $^{10}$, H.M. Zhang ${ }^{10}$, H.Y. Zhang ${ }^{22}$, J.L. Zhang ${ }^{17}$, J.W. Zhang ${ }^{9}$, L.X. Zhang ${ }^{11}$, Li Zhang ${ }^{20}$, Lu Zhang ${ }^{14}$, P.F. Zhang ${ }^{20}$, P.P. Zhang ${ }^{14}$, R. Zhang ${ }^{7,13}$, S.R. Zhang ${ }^{14}$, S.S. Zhang ${ }^{1,3}$, X. Zhang ${ }^{10}$, X.P. Zhang ${ }^{1,3}$, Y.F. Zhang ${ }^{8}$, Y.L. Zhang ${ }^{1,3}$, Yi Zhang ${ }^{1,13}$, Yong Zhang ${ }^{1,3}$, B. Zhao $^{8}$, J. Zhao ${ }^{1,3}$, L. Zhao ${ }^{6,7}$, L.Z. Zhao ${ }^{14}$, S.P. Zhao ${ }^{13,22}$, F. Zheng ${ }^{33}$, Y. Zheng ${ }^{8}$, B. Zhou ${ }^{1,3}$, H. Zhou ${ }^{28}$, J.N. Zhou ${ }^{15}$, P. Zhou ${ }^{10}$, R. Zhou ${ }^{9}$, X.X. Zhou ${ }^{8}$, C.G. Zhu ${ }^{22}$, F.R. Zhu ${ }^{8}$, H. Zhu ${ }^{17}$, K.J. Zhu ${ }^{1,2,3,6}$ and X. Zuo ${ }^{1,3}$

${ }^{1}$ Key Laboratory of Particle Astrophyics \& Experimental Physics Division \& Computing Center, Institute of High Energy Physics, Chinese Academy of Sciences, 100049 Beijing, China.

${ }^{2}$ University of Chinese Academy of Sciences, 100049 Beijing, China.

${ }^{3}$ TIANFU Cosmic Ray Research Center, Chengdu, Sichuan, China.

${ }^{4}$ Dublin Institute for Advanced Studies, 31 Fitzwilliam Place, 2 Dublin, Ireland.

${ }^{5}$ Max-Planck-Institut for Nuclear Physics, P.O. Box 103980, 69029 Heidelberg, Germany.

${ }^{6}$ State Key Laboratory of Particle Detection and Electronics, China.

${ }^{7}$ University of Science and Technology of China, 230026 Hefei, Anhui, China.

${ }^{8}$ School of Physical Science and Technology \& School of Information Science and Technology, Southwest Jiaotong University, 610031 Chengdu, Sichuan, China.

${ }^{9}$ College of Physics, Sichuan University, 610065 Chengdu, Sichuan, China.

${ }^{10}$ School of Astronomy and Space Science, Nanjing University, 210023 Nanjing, Jiangsu, China.

${ }^{11}$ Center for Astrophysics, Guangzhou University, 510006 Guangzhou, Guangdong, China.

${ }^{12}$ School of Physics and Technology, Wuhan University, 430072 Wuhan, Hubei, China.

${ }^{13}$ Key Laboratory of Dark Matter and Space Astronomy, Purple Mountain Observatory, Chinese Academy of Sciences, 210023 Nanjing, Jiangsu, China.

${ }^{14}$ Hebei Normal University, 050024 Shijiazhuang, Hebei, China.

${ }^{15}$ Key Laboratory for Research in Galaxies and Cosmology, Shanghai Astronomical Observatory, Chinese Academy of Sciences, 200030 Shanghai, China.

${ }^{16}$ Key Laboratory of Cosmic Rays (Tibet University), Ministry of Education, 850000 Lhasa, Tibet, China.

${ }^{17}$ National Astronomical Observatories, Chinese Academy of Sciences, 100101 Beijing, China.

${ }^{18}$ School of Physics and Astronomy \& School of Physics (Guangzhou), Sun Yat-sen University, 519000 Zhuhai, Guangdong, China.

${ }^{19}$ Dipartimento di Fisica dell'Università di Napoli ‘Federico II”, Complesso Universitario di Monte Sant’Angelo, via Cinthia, 80126 Napoli, Italy.

${ }^{20}$ School of Physics and Astronomy, Yunnan University, 650091 Kunming, Yunnan, China.

${ }^{21}$ D'epartement de Physique Nucl'eaire et Corpusculaire, Facult'e de Sciences, Universit'e de Gen 'eve, 24 Quai Ernest Ansermet, 1211

Geneva, Switzerland.

${ }^{22}$ Institute of Frontier and Interdisciplinary Science, Shandong University, 266237 Qingdao, Shandong, China.

${ }^{23}$ Department of Engineering Physics, Tsinghua University, 100084 Beijing, China.

${ }^{24}$ School of Physics and Microelectronics, Zhengzhou University, 450001 Zhengzhou, Henan, China.

${ }^{25}$ Institute for Nuclear Research of Russian Academy of Sciences, 117312 Moscow, Russia. 
${ }^{26}$ School of Physics, Peking University, 100871 Beijing, China.

${ }^{27}$ School of Physical Science and Technology, Guangxi University, 530004 Nanning, Guangxi, China.

${ }^{28}$ Tsung-Dao Lee Institute \& School of Physics and Astronomy, Shanghai Jiao Tong University, 200240 Shanghai, China.

${ }^{29}$ Yunnan Observatories, Chinese Academy of Sciences, 650216 Kunming, Yunnan, China.

${ }^{30}$ Department of Physics, Faculty of Science, Mahidol University, 10400 Bangkok, Thailand.

${ }^{31}$ Moscow Institute of Physics and Technology, 141700 Moscow, Russia.

${ }^{32}$ Key Laboratory of Radio Astronomy, Purple Mountain Observatory, Chinese Academy of Sciences, 210023 Nanjing, Jiangsu, China.

${ }^{33}$ National Space Science Center, Chinese Academy of Sciences, 100190 Beijing, China. 